\title{
Effects of Sucrose on Germination and Seedling Development of Brassica Napus
}

\author{
Feng Xu \\ Institute of Life Sciences, Jiangsu University \\ Zhenjiang 212013, China \\ E-mail: xufeng19860707@yahoo.com.cn \\ Xiaoli Tan (Corresponding author) \\ Institute of Life Sciences, Jiangsu University \\ Zhenjiang 212013, China \\ Tel: 86-511-8291-4326_E-mail: xltan@ujs.edu.cn \\ Zheng Wang \\ Institute of Life Sciences, Jiangsu University \\ Zhenjiang 212013, China \\ E-mail: wangzheng4466@163.com
}

The research is financed by the student research project of Jiangsu University (No. 08A183).

\begin{abstract}
Sucrose is the major carbon form translocated in higher plants, and also works as an important signaling molecule that regulates genes involved in photosynthesis, metabolism, and developmental processes. Many studies focused on glucose, normal decomposed product of sucrose, demonstrated that glucose resulted in a delay of germination and an inhibition of seedling development. In this study, we determined the effects of sucrose on germination kinetics and seedlings development. Our results indicated that exogenous sucrose didn't have a similar role to glucose, showed $167 \mathrm{mM}$ sucrose delayed the rate of seed germination in wild-ecotype seeds by extending peak time of germination, and had multiple roles to seedling development. Overhigh concentration of sucrose $(333 \mathrm{mM})$ restrained germination and seedling development seriously. Further experiments of transcript profiles of genes involved in energy and carbohydrate metabolism indicated that seedlings absorbed and utilized the exogenous sucrose as a priority while reduced the need for internal source.
\end{abstract}

Keywords: Brassica napus, Sucrose, Germination, Seedling

\section{Introduction}

Sucrose is an important carbohydrate in most plants. It has multiple functions such as regulating photosynthesis and respiration, serving as storage compound and helping to maintain the osmotic pressure in the cytosol (Rita Teresa Teixeira, 2005).In oilseeds, sucrose is major carbohydrate transport form upon seeds germination and seedling development.

During germination and seedling development, storage triacylglycerols are cleaved by lipases from their glycerol backbone in the oil body, and convert to carbohydrates major as sucrose for transport to the root and shoot axes (Eastmond, 2006). Sucrose is used as a substrate for biosynthesis and is respired for energy. After being transported to the glyoxysome, sucrose is degraded by beta-oxidation to acetyl-CoA. The glyoxylate cycle ultimately catalyzes the condensation of two of these acetyl-CoA molecules to form succinate, which is then transported to the mitochondrion and metabolized by a partial TCA cycle (Kimberly L. Falk, 1998).

Sucrose also functions as an important signaling molecule in the regulation of germination and seedling development although sucrose is readily converted into fructose and glucose. Combined treatments of glucose and fructose were less 
effective than sucrose treatments (Francisco Arenas-Huertero, 2000), suggesting that sucrose functions as a signaling molecule.

The sucrose-mediated translational control mechanism is an important sucrose-dependent regulation of mRNA translation. Sucrose acts as a signaling molecule in the control of translation of the S1 class basic leucine zipper transcription factor bZIP (Hummel M, 2009). The main bZIP open reading frames (ORFs) are preceded by upstream open reading frames (uORFs), which have general function of Sucrose-induced repression of translation (SIRT) (Anika Wiese, 2004). Arabidopsis homologues of ATB2/AtbZIP11, which harbour the conserved uORF, also show SIRT(Wiese A, 2005). AtbZIP11 is translational repressed in response to sucrose. Target genes of such transcription factors will then be regulated in sucrose-dependent way, resulting in sucrose-regulated changes in metabolism (Rahmani F, 2009).

Previous study always focused on glucose, which illustrated that glucose negatively affected seed germination and early seedling development. Sucrose is an important carbon source for development in plants and wildly used in plant culture. So we evaluated the role of sucrose on germination and seedling development of Brassica napus. Our findings suggested that extraneous sucrose influenced activity of seedling metabolism dramatically, both as metabolite and singalling molecular in Brassica napus, and regulated expression of the genes involved in energy and carbohydrate metabolisms.

\section{Materials and methods}

\subsection{Growth of Plant Material and measurement of developmental conditions}

Brassica napus seeds were surface-sterilized and water-imbibed in the dark for $2 \mathrm{~d}$ at $4{ }^{\circ} \mathrm{C}$. Seeds were transferred to $1 \times$ Murashige and Skoog medium, sucrose was also added where indicated. The plant material was incubated in the dark at $4^{\circ} \mathrm{C}$ for $2 \mathrm{~d}$ to break dormancy and then was transferred to light at $24^{\circ} \mathrm{C}$. All cultures were grown with a photoperiod of $16 \mathrm{~h}$ of light and $8 \mathrm{~h}$ of dark. Germination kinetics was determined by measuring the time of radicle emergence from repeated experiments with duplicate plates of approximately 80 seeds, and selected the seeds germinating in the same period to measure the length of hypocotyls and roots. to be used for phenotype analyses.

\subsection{RNA Extractions and Reverse transcription}

The whole developing seedlings were sampled at 2, 4 and 6 days after germination (DAG), respectively, and stored at $-70^{\circ} \mathrm{C}$ for RNA isolation. Total RNA was extracted by Trizol (invitrogen) reagent. The RNA was quantitated by A260 measurements and by ethidium bromide staining intensity and then stored as an ethanol precipitate at $-20^{\circ} \mathrm{C}$ until use. cDNA was synthesized using $2 \mu \mathrm{g}$ of RNA from each treatment by M-MLV Reverse Transcriptase (Takara, Japan) according to the manufacture's protocol.

\subsection{Semi-quantitative reverse transcription ( $R T)$-PCR analysis}

For RT-PCR assays, the cDNAs of different treatments were used as template. The primers sequences are shown in Table 1. The PCR were performed as following procedure: Denatured at $95^{\circ} \mathrm{C}$ for 4 min followed by 35 cycles $\left(95^{\circ} \mathrm{C}\right.$ for $35 \mathrm{~s}, 56^{\circ} \mathrm{C}$ for $40 \mathrm{~s}$ and $72^{\circ} \mathrm{C}$ for $1 \mathrm{~min}$ ). The same cDNAs were also used to amplify the Actin as an internal control. $10 \mu \mathrm{l}$ products of each reaction were electrophoresed on $1 \%$ agarose gel stained with ethidium bromide.

\section{Results}

\subsection{Exogenous sucrose delay the seed germination of Brassica napus}

As degraded product of sucrose, glucose caused ABA accumulation, which resulted in a delay of germination in wide levels of concentration (John Price, 2003). As our results showed, exogenous sucrose also caused a delay of germination like glucose (Figure1). We compared germination kinetics with intermediate $(167 \mathrm{mM})$, high $(333 \mathrm{mM})$ concentration of sucrose. The difference from glucose was that seeds treated with intermediate concentration of sucrose $(167 \mathrm{mM}$ sucrose) and WT seeds reached the peak time of germination at the same time, while seeds treated with $167 \mathrm{mM}$ sucrose led to the extension of active phase of germination. What was similar to the seeds treated with glucose was that high concentration of glucose inhibited the seeds from germinating seriously and resulted in a delay of germination peak time (figure2).

\subsection{Exogenous sucrose has multiple roles on seedling development}

It has been suggested that availability of abundant glucose can exert a profound influence, resulting in seedling developmental arrest(Jang JC, 1997).Similar to glucose, High levels of exogenous sucrose inhibited seedlings development seriously, and this effect could not be mimicked by an osmotic effect (John Price, 2003). Our results showed that in Brassica napus, the development of hypocotyls was inhibited (figure 3) while roots were promoted (figure 4) in the medium containing $167 \mathrm{mM}$ sucrose, which was different from the pattern in Arabidopsis.

\subsection{Exogenous sucrose regulates expression of the key genes involved in metabolism}

Exogenous sucrose as an environmental factor greatly impacted plant gene expression and concentrations of cellular 
metabolites (Solfanelli C, 2006; Hummel M, 2009). In this study, expression profiles of genes involved in energy metabolism in Brassica napus were analyzed (Figure 5). Hexokinase (HXK) and pyruvate dehydrogenase (PDH) are rate limited enzymes in glycolysis, HXK also plays a central role as a conserved glucose sensor in sugar signaling pathway (John Price, 2003). Citrate synthase (CS) is the first enzyme of the tricarboxylic-acid cycle. ADP-glucose pyrophosphorylase (ADG) is a key-enzyme of starch synthesis in plant. Glyoxysomal Isocitrate lyase (GIL) is unique to the glyoxylate cycle. As to the control treated without sucrose, each gene had the similar transcription profile, and attended to the top point at the $4 \mathrm{DAG}$, then decreased. Surprisingly, exogenous sucrose affected the expression of all genes strongly. Collectively, exogenous sucrose restrained expression of all these genes. Transcripts of $B n H X K$ and $B n P D H$ began to accumulate at the 2 DAG. Conversely, $B n C S, B n A D G$ and $B n G I L$ gradually decreased. It's suggested that exogenous sucrose influenced the energy and carbohydrate metabolism of the seedling dramatically.

\section{Discussion}

Our results indicated that germination and seedling development of Brassica napus was regulated by sucrose, and sucrose has more complicated roles compared with glucose. For the seedlings of Brassica napus, exogenous $167 \mathrm{mM}$ sucrose only inhibited the hypocotyls growth but promoted the roots, while exogenous $167 \mathrm{mM}$ glucose caused ABA accumulation and inhibited seedling development in Arabidopsis (John Price, 2003). Our results implied that Sucrose promoted the roots growth probably because the sucrose was absorbed and used as carbon source by the roots, which improved growth of roots by counteracting negative regulating of sucrose signaling. However, the hypocotyls couldn't absorb exogenous sucrose, and the growth was inhibited by the sugar signaling. As for this process, whether the ABA work as an important signaling molecule is not clear.

Transcript profiles also indicated that sucrose play important roles in seedling developments. Compared to the controls, the expression of genes involved in metabolism in seedling treated with sucrose had been inhibited obviously and had complicated alterations. These results suggested that the seedlings absorbed and utilized the exogenous sucrose as a priority while reduced the need for internal source. The decreased expressions of BnCS, BnADG and BnGIL indicate the depression of the gluconeogenesis. While the expression of $B n P D H$ and $B n H X K$ related with catabolism which take sugar as their substrate (mainly glucose) increased gradually. Glucose, the decomposed products of sucrose, might play an important role in this regulation. It also has been reported that glucose affect the expression of a diverse array of genes involved in carbohydrate metabolism (Wenyan Xiao, 2000; John Price, 2003),

\section{References}

Anika Wiese, N.E., Barry Wobbes, \& Sjef Smeekens. (2004). A Conserved Upstream Open Reading Frame Mediates Sucrose-Induced Repression of Translation. The Plant Cell, 16, 1717-1729.

Eastmond, P.J. (2006). SUGAR-DEPENDENT1 Encodes a Patatin Domain Triacylglycerol Lipase That Initiates Storage Oil Breakdown in Germinating Arabidopsis Seeds. The Plant Cell, 18, 665-675.

Francisco Arenas-Huertero, A.A., Li Zhou, Jen Sheen, \& Patricia León. (2000). Analysis of Arabidopsis glucose insensitive mutants, gin5 and gin6, reveals a central role of the plant hormone ABA in the regulation of plant vegetative development by sugar. Genes Dev, 14(16), 2085-2096.

Hummel M, R.F., Smeekens S, \& Hanson J. (2009). Sucrose-mediated translational control. Ann Bot (Lond), 104(1), $1-7$.

Jang JC, S.J. (1997). Sugar sensing in higher plants. Trends Plant Sci, 115, 208-213.

John Price, T.-C.L., Shin Gene Kang, Jong Kuk Na, \& Jyan-Chyun Jang. (2003). Mechanisms of Glucose Signaling during Germination of Arabidopsis. Plant Physiology, 132, 1-15.

Kimberly L. Falk, R.H.B., Chengbin Xiang, \& David J. Oliver. (1998). Metabolic Bypass of the Tricarboxylic Acid Cycle during Lipid Mobilization in Germinating Oilseeds. Plant Physiology, 117, 473-481.

Rahmani F, H.M., Schuurmans J, Wiese-Klinkenberg A, Smeekens S, \& Hanson J. (2009). Sucrose control of translation mediated by an upstream open reading frame-encoded Peptide. Plant Physiology, 150(3), 1356-1367.

Rita Teresa Teixeira, \& C.K.a.K.G. (2005). Modified sucrose, starch, and ATP levels in two alloplasmic male-sterile lines of B. napus. Journal of Experimental Botany, 56(414), 1245-1253.

Solfanelli C, P.A., Loreti E, Alpi A, \& Perata P. (2006). Sucrose-specific induction of the anthocyanin biosynthetic pathway in Arabidopsis. Plant Physiology, 140, 637-646.

Wenyan Xiao, J.S., \& Jyan Chyun Jang. (2000). The role of hexokinase in plant sugar signal transduction and growth and development. Plant Molecular Biology, 44, 451-461.

Wiese A, E.N., Wobbes B, \& Smeekens S. (2005). Sucrose-induced translational repression of plant bZIP-type transcription factors. Biochem Soc Trans, 33, 272-275. 
Table 1. Oligonucleotide primers used in RT-PCR analysis

\begin{tabular}{llll}
\hline Genes & $\begin{array}{l}\text { Accession } \\
\text { numbers }\end{array}$ & Primer & Nucleotide sequence \\
BnGIL & Y13356 & F & 5'-ATCCATACGATACCGTTCCTAA \\
& & R & 5'- TCATCTGCTGATCCGTCATACC \\
\multirow{2}{*}{ BnHXK } & AF454961 & F & 5'-CTCGGTGGCAAGCAAGAC \\
& & R & 5'- GCATAGCAGACATGGTAGGA \\
BnPDH & ES902002 & F & 5'- TTACGGGGGCTCCTACAA \\
& & R & 5'-ACACTCCTCCACTATCAAAACC \\
BnCS & ES900694 & F & 5'-GTATCAGGTCCCAGTCTCGG \\
& & R & 5'-CAACACCACTAGAGGCAAGA \\
BnADG & AJ271162 & F & CGTCCGCAAAACTCTTCAAC \\
& & R & TCTATCTCCTGCTTTCTCGC \\
Actin & AY570244 & F & 5'-ATGGCCGATGGTGAGGACATTC \\
& & R & 5'-GGTGCGACCACCTTGATCTTC \\
\hline
\end{tabular}

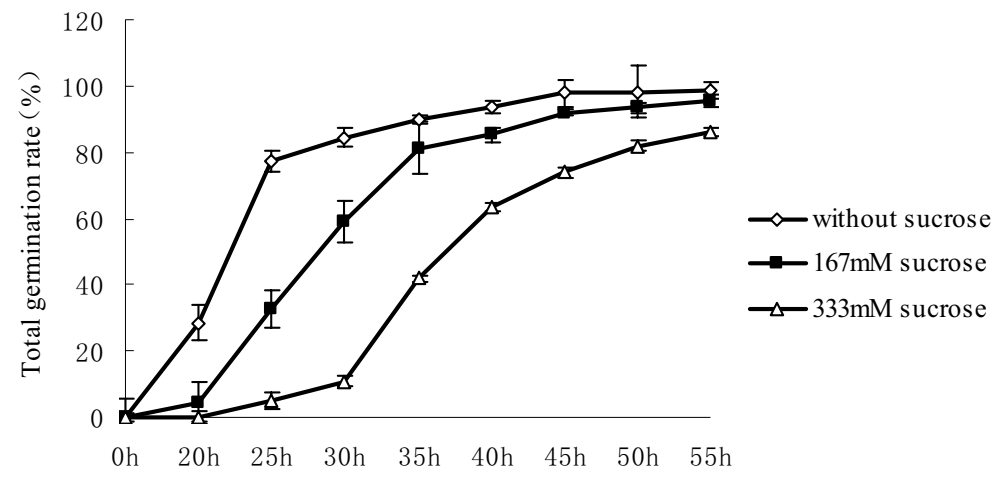

Figure 1. Exogenous sucrose resulted in a delay of germination as glucose. Brassica napus seeds were surface-sterilized and water-imbibed in the dark for $2 \mathrm{~d}$ at $4^{\circ} \mathrm{C}$. Seeds were transferred to Murashige and Skoog plates without sucrose or with sucrose (concentration indicated in the figure). The plant material was incubated in the dark at $4^{\circ} \mathrm{C}$ for $2 \mathrm{~d}$ to break dormancy and was then transferred to light at $24^{\circ} \mathrm{C}$. Germination kinetics was determined by measuring the time of radicle emergence after transfer to constant light and $24^{\circ} \mathrm{C}$.

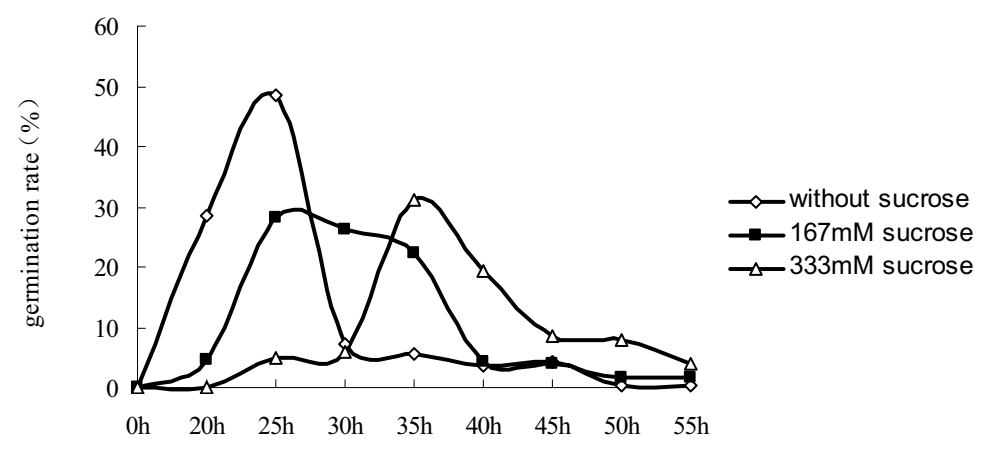

Figure 2. Compared with control treated without sucrose, $167 \mathrm{mM}$ sucrose treatment caused the extension of peak time of germination, while $333 \mathrm{mM}$ sucrose resulted in a delay of peak time of germination as glucose. 


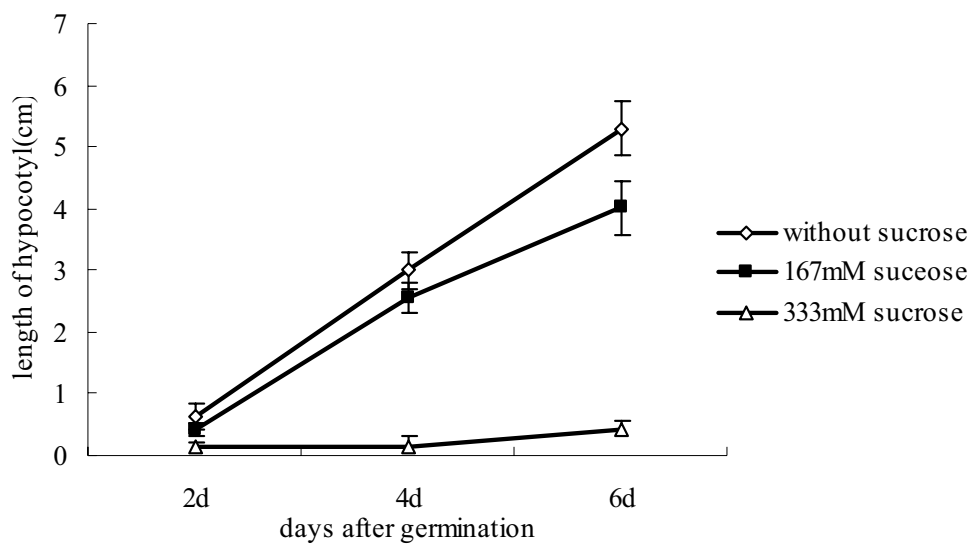

Figure 3. The development of hypocotyls was inhibited in Murashige and Skoog plates containing sucrose, especially in the medium with $333 \mathrm{mM}$ sucrose, and this effect could not be mimicked by an osmotic effect.

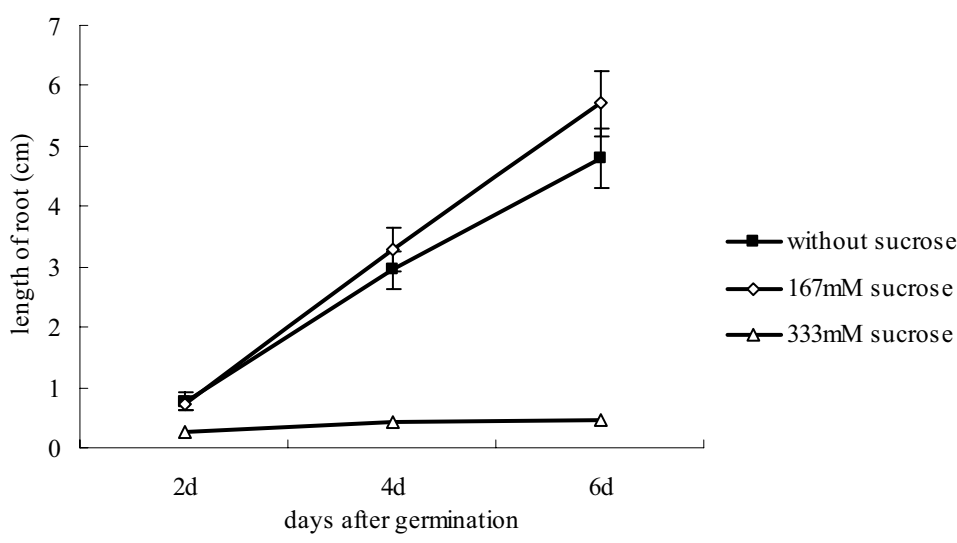

Figure 4. Contrast to hypocotyls, development of roots was promoted in Murashige and Skoog plates containing $167 \mathrm{mM}$ sucrose. While in the medium with $333 \mathrm{mM}$ sucrose, development of roots was still inhibited strongly.

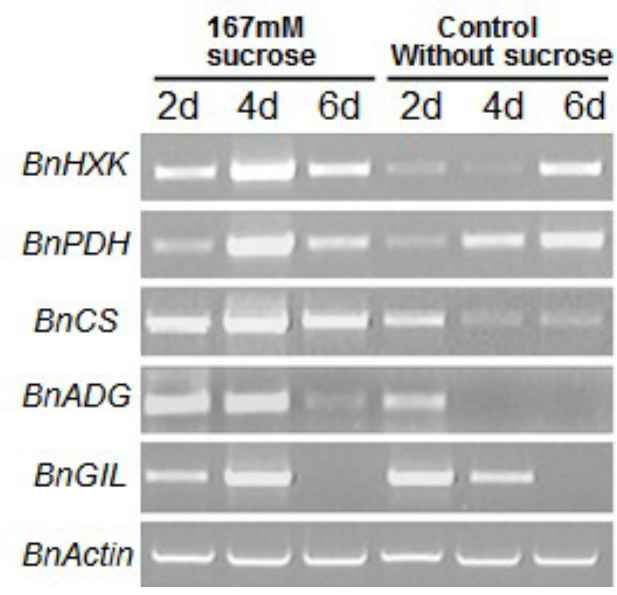

Figure 5. Exogenous sucrose regulated expression of the key genes involved in metabolism. As to the control treated without sucrose, each gene had the similar transcription profile, and at the 4 DAG attends to the top point, then decreases. Surprisingly, exogenous sucrose affected the expression of all genes strongly. Transcripts of $B n H X K$ and $B n P D H$ began to accumulate at the 2DAG, conversely, BnCS, BnADG and BnGIL gradually decreased. Generally speaking, exogenous sucrose restrained expression of all genes. 